\title{
WEIGHTINESS OF THE DISPERSIVE RATE IN STOCHASTIC ACCELERATION PROCESSES
}

\author{
J. Pérez-Peraza \\ Instituto de Geofísica, UNAM, 04510 C.U., Mexico D.F., Mexico \\ AND \\ A. GALlegos-CRUZ ${ }^{1}$ \\ UPIICSA, IPN, Te 950, 08400 Mexico D.F., Mexico \\ Received 1993 July 15; accepted 1993 August 17
}

\begin{abstract}
We present analytical solutions to the transport equation of the accelerated particles that are valid over the entire energy range (nonrelativistic, transrelativistic, and ultrarelativistic) for the time-dependent and equilibrium cases. On the basis of these overall spectra, we have studied the relative importance of the term of average (systematic) energy increase and the term of diffusion (spread) in energy that define the evolution of accelerated particles within the frame of a simplified diffusion-convection transport equation, when the particle distribution function $N(E, t)$ is assumed to be independent of spatial pitch-angle diffusion (isotropic). Since in some astrophysical works, only the term of average energy increase in the simplified transport equation in energy space is considered to be leading directly to a power-law-type spectrum, we have established the conditions under which such an approximation may be justified. The analysis is illustrated for acceleration by two different kinds of turbulence: MHD and Langmuir waves. The omission of the term of energy spread leads, in general, to an important depletion or overproduction of the accelerated particle flux that must be seriously considered in any calculation of the flux of secondary radiation produced by the accelerated particles. The presented analytical spectra may be of particular relevance to gamma-ray, neutron, and pion production in solar flares as well as in other astrophysical sites.
\end{abstract}

Subject headings: acceleration of particles - MHD — Sun: general - turbulence - wave motions

\section{INTRODUCTION}

When turbulence is excited in a plasma, random electric fields are produced in a natural way, so that the interaction of these turbulent fields with the plasma particles is of stochastic nature: in such a turbulent plasma, charged particles may undergo resonant elemental interactions with the fields of the turbulent oscillations leading to small stochastic changes in particle energy. These resonant wave-particle interactions occur under particular conditions of coherence between the properties of the waves (frequency, wave-length, phase velocity) and the properties of particles (velocity and gyrofrequency). Particles may absorb energy from the turbulent fields, or cede energy to the plasma oscillations, so that particle acceleration, plasma heating, or wave growth may take place.

Since each individual particle gains or loses energy through small energy change steps, wave-particle resonant interactions may be seen as independent events among them. This behavior allows the study of the evolution of an accelerated particle flux from the statistical point of view, by defining distribution functions for the interacting particles and wave turbulence, and deriving the corresponding transport equation. In general, the problem is reduced to studying how the distribution function of the plasma particles changes under the influence of turbulent waves, and how these oscillations are excited or damped by a given particle distribution, although most works deal with the former feature. Since the density of the resonant

\footnotetext{
'Doctoral student at the UAM-Iztapalapa, México D.F., Mexico.
}

accelerated particles is relatively low, their mutual binary collisions are disregarded, so that acceleration is studied as a collisionless problem. To study the influence of oscillations on the evolution of the distribution of the accelerated particles, the transport equation may be derived in several ways (e.g., Jones 1992; Schlickeiser 1992). Within the frame of strong turbulence, particle evolution is usually studied by MHD approximations (e.g., Priest 1984), or by a renormalization approach (Byakov 1992). Within the context of weak turbulence it is assumed that the energy gain per resonant collision is smaller than the particle energy, so that stochastic acceleration is seen as a diffusion process in energy space: the most common methods to derive the transport equation are based on the Chapman-Kolmogorov equation, and on the collisionless Boltzmann-Vlasov equation. The former is based on the calculation of expectation values and mean square deviations of particle momentum and position along the unperturbed particle trajectories, and the latter is based on a quasi-linear formalism, which does not consider nonlinear effects, such as wave-wave interactions, but only takes into account the effects of induced Cherenkov (and/or cyclotron) absorption and emission of waves by the resonant particles. The basic assumption is that the effect of wave-particle interaction on the orbit of the particles can be treated as a perturbation, by considering the parameters of the particle distribution function as an average part plus a small fluctuating part. Under certain grounds, both formalisms lead to a Chandrasekhar equation. The latter adapted to the specific case of the generation of energetic cosmic particles may be reduced to a Fokker-Planck-type equation (Schatzman 1966). 
Derivation of the transport equation from the Vlasov equation usually has been done under a certain number of simplifications such as isotropy and homogeneity in space and time of wave turbulence and isotropy of particles during their acceleration, so the particle phase space density $f(p, r, t, \mu)$ describing the distribution function of particles of momentum $p$, position $r$, and cosine of particle pitch angle $\mu=p_{l} / p$ at time $t$ becomes a pitch-angle averaged particle distribution $f(p, t)$, and the Fokker-Planck coefficient related to spatial diffusion is dropped out. A resulting equation known as the diffusive-convection transport equation is usually employed under oversimplified versions to describe the evolution of energetic cosmic particles.

At present, stochastic acceleration within the context of the quasi-linear approximation is worked out equivalently, by either a diffusion equation in momentum space, or by a FokkerPlanck-type equation in energy space: the diffusion transport equation is characterized by a momentum diffusion coefficient $D(p)$, whereas the Fokker-Planck equation contains both diffusion and convection coefficients. The latter more conspicuously shows the convective and diffusive nature of the energization process, and since energetic particle data are generally described in terms of kinetic energy, this approach is most often employed for semiquantitative purposes. By introducing source and sink effects in the diffusion-convection equation and neglecting adiabatic energy changes and competitive energy loss processes during acceleration, the equation is reduced to the following expression:

$$
\begin{aligned}
\frac{\partial N(E, t)}{\partial t} & +\frac{\partial}{\partial E}[A(E) N(E, t)] \\
& -\frac{1}{2} \frac{\partial^{2}}{\partial E^{2}}[D(E) N(E, t)]+\frac{N(E, t)}{\tau(E, t)}=Q(E, t),
\end{aligned}
$$

where $N(E, t)=\left(4 \pi p^{2} / v\right) f(p, t), v=$ particle velocity, and $A(E)=\langle d E / d t\rangle=\frac{d}{d t}\langle E\rangle=\left(1 / p^{2}\right) \frac{\partial}{\partial p}\left[v p^{2} D(p)\right]$ is the rate at which the average energy $\langle E\rangle$ per particle increases. This is usually designated as the Fokker-Planck coefficient of convection, or as the rate of systematic acceleration. $D(E)=\left\langle d E^{2}\right\rangle$ $d t\rangle=2 v^{2} D(p)$ is the rate at which the variance in particle energy $\left\langle(E-\langle E\rangle)^{2}\right\rangle$ increases and is generally known as the Fokker-Planck coefficient for diffusion in energy, or as the rate of dispersive acceleration, or even the rate of fluctuational acceleration (e.g., Tsytovich 1977; Melrose 1980). The source term, $Q(E, t)$, denotes particle injection into the acceleration process, and $\tau^{-1}$ is the probability of particle disappearance from the acceleration volume by escape or nuclear transformation. $D(p)=\langle(\Delta p)\rangle^{2} / \Delta t$ describes the effect that plasma turbulence has on particles when $\Delta p \ll p$.

In this paper we will deal with the following tasks: (1) We develop some previous results by Gallegos-Cruz \& PérezPeraza (1991), concerning the analytical solution of equation (1), based on the WKBJ approximation method. We present approximate analytical solutions valid through the entire energy range, including the transrelativistic range, for both the time-dependent and the stationary solutions. (2) With the derived energy spectra we determine how much information is lost by neglecting the dispersive rate in equation (1), as is some times done in the literature (e.g., Ginzburg \& Syrovatskii
1964; Cheng 1972; Heristchi et al. 1976; Pikel'ner \& Livishts 1977; Eichler 1979; Vilmer et al. 1982, 1986; MacKinnon et al. 1983). (3) We attempt to establish the range of acceleration parameters in solar source conditions under which the neglect of the dispersive term does not introduce an important deviation from the actual particle energy spectrum; that is, whether the diffusive fundamental essence of a stochastic process may be substituted by the secular behavior of an average systematic acceleration rate.

\section{DIFFUSIVE AND CONVECTIVE ACCELERATION}

Although stochastic acceleration is essentially a diffusion process in energy space, the mathematical split of the transport equation into two terms, the convective and diffusive, may be interpreted as follows: in spite of the statistical nature of the diffusion process that characterizes the wave particle energy interchange, a net energy gain tendency is established, which may be seen as an average rate of deterministic nature $\langle d E /$ $d t\rangle$. However, there is a spread in the energy change around the average energy change rate, which results in a dispersion of the energy reached by particles. It is precisely because of the stochastic nature of the elementary accelerating interactions that the energy change $\Delta E$ is not necessarily the same for particles with the same initial energy (some of them may even decrease their energy in some kind of interactions). Such a statistical energy dispersion is described as an energy diffusion effect and determined by the variance of particle energy at a rate $\left\langle d E^{2} / d t\right\rangle$.

In plasma physics it is frequently argued that certain types of turbulence are ineffective in accelerating particles because only energy diffusion takes place, and this is translated into plasma heating. Indeed in $r f$ heating of fusion plasmas only the energy-spread effect is important (Stix 1975). Consequently, in cosmic-ray physics the relative importance of the dispersive and the systematic rates for particle acceleration has been sometimes underestimated and discussed rather on qualitative basis, for the specific case of $\beta=(v / c) \cong 1$, with a clear tendency to neglect the former relative to the latter (Tystovich 1966; Melrose 1968, p. 213). Under such a tendency it is quite frequent to find in the literature energy spectra derived from the Fokker-Planck equation, when one only considers the systematic energy gain rate (Ginzburg \& Syrovatskii 1964; MacKinnon et al. 1983; Vilmer et al. 1982, 1986). In fact, the original statistical mechanism of Fermi (1949) only considered the systematic acceleration rate. Davis (1956) and Parker \& Tidman (1958) were the first to emphasize the diffusive nature of stochastic acceleration.

Even if the average systematic energy gain is null, energetic particles may be accelerated by the effect of energy spread. Schatzman (1966) derived the flux of particles when the average energy loss balances the average energy gain of the Fermi mechanism, so that only diffusion in energy accounts for particle acceleration. According to Lacombe \& Manganey (1969), acceleration of nonrelativistic solar particles by ion-sound waves is determined only by energy diffusion. In this context, Tsytovich (1970) has shown that for some kind of turbulence, as ion-acoustic oscillations, the systematic acceleration rate falls off rapidly for particle velocities higher than the sound velocity, while the acceleration of relativistic particles by Langmuir waves is determined by the systematic acceleration rate. 
However, for nonrelativistic particles dispersive acceleration is the decisive one, if the spectrum of turbulence does not contain waves whose phase velocity is close to the velocity of particles. On the other hand, Ginzburg \& Syrovatskii (1964) and Melrose (1980) have pointed out that for Fermi acceleration with $D(p)=\alpha p^{2} / 3 \beta$ (where $\alpha$ is the acceleration efficiency parameter) the ratio of the secular rate to the dispersive rate, when $\beta \cong 1$, is of the order of $4: 1$. However, it is worthwhile to emphasize that such a ratio is meaningless because it refers only to the numerical coefficients of the rates, and in no way to the ratio of the corresponding convective and diffusive complete terms of the left-hand side of equation (1), that under the nonrelativistic regime their dependence on $E$ and on $\beta$ differs drastically from each other. We claim in this work that for a correct quantitative evaluation of the effect of both rates on the number density of the accelerated particles and their energy distribution, equation (1) needs to be solved. It must be realized that the relative effect of both terms cannot be generalized, but it may vary greatly, depending on the assumptions made to solve equation (1), regarding the scenario for the acceleration phenomenon, that is, the acceleration process, the turbulence spectrum, and the spectrum of the initial particle population.

In a preliminary analysis, Gallegos-Cruz \& Pérez-Peraza (1987) have shown that even in the extreme case when $\beta \cong 1$, the contribution of the dispersive term in equation (1) does not result in mere fluctuations in particle number density, but it represents an important energy-dependent effect of particle overproduction or depression and may be, in some cases, the determinant factor of particle production. The analysis was extended by Pérez-Peraza \& Gallegos-Cruz (1994) to any $\beta$ value, for the particular case of the Fermi classic mechanism, that is, elastic collisions with hard spheres of size scale larger than particle gyroradius (large-amplitude compressions). Here the analysis is generalized to the case of turbulent resonant acceleration, by illustrating it with two kinds of turbulent oscillations susceptible of existing in solar particle sources, Langmuir and MHD waves.

\section{SCENARIO AND ACCELERATION RATES}

Solution of the transport equation (1) requires the establishment of the Fokker-Planck coefficients. Calculation of such coefficients must be done within the frame of specific plasma wave modes in a given physical system. Convective and diffusive Fokker-Planck coefficients for low- and high-frequency MHD modes are fairly well known in the literature, as they have been derived in terms of the pitch-angle-averaged diffusion coefficient $D(p)$, (e.g., Tverskoi 1967; Kulsrud \& Ferrari 1971; Tsytovich 1977; Melrose 1980, 1986). For weak turbulence, the quasi-linear coefficients of Alfvén waves for all pitch angles, parallel and oblique waves, and both directions and polarization states have been derived by Schlickeiser (1989), Dung \& Schlickeiser (1990), and Steinacker \& Miller (1992). However, in this work we do not require such deep detail, and it is enough to keep in mind that in the linear wave-particle resonance involving MHD turbulence, $D(p) \sim p^{n} / \beta$, for Landau-Cherenkov resonance, or $D(p) \sim R^{n} / \beta$ for gyroresonance ( $R=[c / Z e] p$ is the particle magnetic rigidity), so that in general $D(p)=D^{*} p^{n} / \beta$, where $D^{*}$ contains the information about the specific acceleration efficiency, $\alpha$, of a given MHD wave mode. For simplicity we consider the case when $n=2$, in such a way that the resonant stochastic acceleration is reduced to a Fermi-like process, involving rather the magnetosonic and Alfvén modes. It should be emphasized that MHD turbulence is thought to be present in energetic particle sources, either for pitch-angle isotropization of particles (short-wavelength waves) or for particle energization by long-wavelength waves (Achterberg 1981), whereas Langmuir turbulence is practically an intrinsic property of turbulent plasmas and may be highly efficient for resonant acceleration of suprathermal particles. The relevance of Langmuir waves for particle acceleration in solar flares has been emphasized by Heyvaerts (1981). The rates of wave-particle energy interchange are derived following the definitions below equation (1): the average convective coefficient describing the systematic energy gain rate is

$$
A(E)=\langle d E / d t\rangle=\zeta \alpha p c=\zeta \alpha \beta \mathscr{E},
$$

where $\mathscr{E}=E+m c^{2}$, and $\zeta=4 / 3$ (but it may take a wide range of values, e.g., Melrose 1980). Similarly, the diffusive coefficient describing the dispersive acceleration rate is

$$
D(E)=\left\langle d E^{2} / d t\right\rangle=(\zeta / 2) \alpha \beta p^{2} c^{2}=(\zeta / 2) \alpha \beta^{3} \mathscr{E}^{2} .
$$

The parameter $\alpha$ depends on the specific MHD turbulence (e.g., Miller et al. 1990), but in this paper $\alpha$ is our free parameter; it depends on the wave number, the total turbulent energy density, and the magnetic energy density, and can roughly be taken as constant. For resonant acceleration by Langmuir oscillations, we have used the momentum diffusion coefficient as given by Tsytovich (1977) and Melrose (1980), so that following the same procedure we obtain

$$
\begin{gathered}
A(E)=\langle d E / d t\rangle=\left(1-0.55 \beta^{2}\right)\left(K \beta^{3 / 2} / \mathscr{E}\right), \\
D(E)=\left\langle d E^{2} / d t\right\rangle=(4 / 9) K \beta^{7 / 2}, \\
K=K_{1} W_{0}=7.45 \times 10^{-2} n_{e} / T^{5 / 4} \mathrm{MeV}^{2} \mathrm{~s}^{-1},
\end{gathered}
$$

where a characteristic Langmuir wave spectrum $W(k)=$ $W_{0} k^{-5 / 2}$ has been assumed (Borosky \& Eilek 1986), and $n_{e}$ is the density of the medium. The energy density content of the turbulence is

$$
W_{0} \sim 4 \times 10^{-9} n_{e}^{9 / 4} / T^{5 / 4} \quad \mathrm{eV} \mathrm{cm}^{-3},
$$

where $T(K)$ is the plasma temperature and $K$ is the free parameter for acceleration by Langmuir turbulence in this work. $W_{0}$ has been evaluated from $R_{u}=\left(U_{\mathrm{tur}} / U_{k T}\right)=10^{-3}\left(n_{e} / T^{3}\right)^{1 / 2}$ (Rose et al. 1987), where $U_{k T}=n_{e} k T$, and $U_{\text {tur }}=(2 /$ 3) $W_{0}\left(v_{T} / \omega_{p}\right)^{3 / 2}$ for the assumed characteristic Langmuir spectrum. Hence $W_{0}$ is obtained from $R_{u}$ with $v_{T}=(2 k T /$ $m)^{1 / 2}$ and $\omega_{p}=\left(4 \pi n_{e} e^{2} / m\right)^{1 / 2}$.

We must remember that effective particle acceleration by the linear wave-particle resonant process requires that particles have a velocity higher than the minimum phase velocity of waves. Although this may be the case of electrons in hot thermal plasma, in general it is not the case for ions and protons. A selective injection process is needed to feed the linear resonant processes with some amount of particles to be accelerated up to relativistic energies. The problem of injection is then narrowly 
related to the scenario of the global energetic particle generation phenomenon. Since the injection problem is of secondary nature for our present goal, we will limit ourselves here to a qualitative discussion. Scenarios for particle production in solar flares pertain to particular events. Attempts to develop a general description of the phenomenon are mostly based on two acceleration phases (e.g., Ramaty 1987): a first phase and second phase associated respectively to the impulsive events and the long-duration X-ray events such as those reported by Cane et al. (1986) and Bai (1986). We assume that solar energetic particle production takes place within the frame of the well-known magnetic bottle model, where the total turbulent energy density $W_{T}$ is enough high for acceleration to take place, whereas when the medium becomes relaxed $\left(W_{T} \sim 0\right)$ we would be dealing with a subsequent transport phase, rather than an acceleration phase. The plasma kinetic pressure may be larger or lower than the magnetic pressure (e.g., PérezPeraza 1986): if the expanding magnetic bottle, containing the source of the flare primary energy release does not open, then a first-phase acceleration event occurs, with some amount of particles drifting out.

If the expanding magnetic bottle opens very quickly, there is essentially a second-phase acceleration event, but if the opening takes long, there is a hybrid event with a first-phase followed by a second-phase acceleration. We assume that in the latter case there is no absolute separation between the phases, and the actual separation is related to the behavior of particles and associated emissions before and after the opening of the bottle. Whatever the kind of flare event, the injection mechanism is closely related to the primary energy release, when all MHD modes, Langmuir waves, and other modes coexist for at least some time after that primary release. For particle supply into the acceleration process we assume here three possibilities: (1) monoenergetic injection at a characteristic suprathermal energy $E_{0},(2)$ a heated thermal spectrum shifted to superAlfvénic velocities, and (3) a preliminary acceleration step by DC fields. In the first two options, we assume that protons and electrons reach super-Alfvénic velocities from nonlinear Landau damping of Alfvén waves (Miller 1991) and gyroresonance of whistlers (Miller et al. 1990), respectively. Alternatively particle heating by quasi-parallel slow magnetosonic waves may be assumed (Gallegos-Cruz et al. 1993), which minimum phase velocity $\left(V_{-}\right)$is much lower than that of fast magnetosonic waves $\left(V_{+}\right)$, so that an important amount of protons have velocity $v \geq V_{-}$, and hence wave energy is distributed not only on electrons but also on protons. For the third injection assumption, we consider particle acceleration associated to the primary energy release in a magnetic neutral current sheet (MNCS), as that given by Priest (1973). Therefore, once particles reach super-Alfvénic velocities, they are accelerated up to ultrarelativistic energies by linear resonant processes, according to the rates given above for MHD and Langmuir turbulence.

For the solution of equation (1) we assume $Q(E, t)=$ $q(E) \Theta(t) \cong q(E)$, where $\Theta(t)$ is the step function. On the other hand, we have also assumed a nondiffusive escape via a leakybox loss term (energy independent $\kappa_{\|}$and averaged $\mu$, so that the characteristic escape time $\tau$ may readily be considered as a constant). Although this assumption can hardly be justified on physical grounds, we have adopted it for the task of comparison of our spectra with the spectra of other authors. Neverthe- less, we illustrate in Appendix $\mathrm{C}$ the analytical steady-state spectrum for a velocity-dependent escape time of the form $\tau \sim$ $1 / \beta$. The later spectrum has the peculiarity that approaches closer an inverse power law than with $\tau=$ constant, which becomes interesting in the light of some recent observational data. We will discuss it elsewhere.

The use of a constant escape time, a time-independent injection, isotropy and homogeneity of particle populations and turbulence, the neglect of energy losses, and averaging over wave numbers, pitch angle, etc., are some of the simplifications that allow us to obtain the solution of the transport equation (1) by approximate analytical methods.

\section{SOLUTIONS TO THE TRANSPORT EQUATION: ENERGY SPECTRA}

The theory known as WKBJ (Wentzel, Kramer, Brilloun, Jeffrey) is a useful tool for solving differential equations of any order, provided they may be linearized. The application of this method for solving analytically the transport equation of accelerated particles at the source level has been reported preliminarily by Pérez-Peraza \& Gallegos-Cruz (1994), and will be extensively described elsewhere. The general solutions to equation (1) (hereafter "complete solutions") and when the term of energy spread in equation (1) is ignored (hereafter "incomplete solutions") for both the time-dependent and the stationary cases are described in the following sections.

\subsection{Complete Solution}

\subsubsection{Analytical Time-dependent Solution}

When both energy changes rates in equation (1) are considered simultaneously, the solution is

$$
\begin{aligned}
& N(E, t)= \frac{D^{1 / 4}(E)}{(4 \pi)^{1 / 2}} \\
& \times \int_{E_{0}}^{E} \frac{\exp \left(-R_{1}\right)}{D^{3 / 4}\left(E^{\prime}\right)}\left[\frac{q_{t=0}\left(E^{\prime}\right)}{t^{1 / 2}} e^{-a t-R_{2} / t}\right. \\
&\left.+(1 / 2)(\pi / a)^{1 / 2} q\left(E^{\prime}\right) R_{3}\left(E^{\prime}, E\right)\right] d E^{\prime} \\
& \text { (particle/energy) }
\end{aligned}
$$

where $R_{1}=(1 / 2) \int_{E^{\prime}}^{E} P_{1} d E^{\prime \prime}$, and $P_{1}=-[(A / D)-(2 / D)(d D /$ $d E)]$, where $E_{0}$ is the threshold injection energy, $R_{2}=(1 / 2)$ $\int_{E^{\prime}}^{E} D^{-1 / 2}\left(E^{\prime \prime}\right) d E^{\prime \prime}, R_{3}=\left[\operatorname{erf}\left(Z_{1}\right)-1\right] \exp \left(2 a^{1 / 2} R_{2}\right)+$ $\left[\operatorname{erf}\left(Z_{2}\right)+1\right] \exp \left(-2 a^{1 / 2} R_{2}\right), Z_{1,2}=(a t)^{1 / 2}+R_{2} t^{-1 / 2}, a=$ $\tau^{-1}+0.5\left[R_{4}(E)+R_{4}\left(E_{0}\right)\right], R_{4}=(d A / d E)-\left(d^{2} D / d E^{2}\right)$, and $R_{4}\left(E_{0}\right)=R_{4}\left(E=E_{0}\right)$.

\subsubsection{Analytical Stationary Solution}

When the term of time evolution in equation ( 1 ) is suppressed, $(\partial N / \partial t)=0$, the solution is

$$
\begin{aligned}
& N(E)=\frac{D^{1 / 4}(E)}{2 a^{1 / 4}(\mathscr{E})} \\
& \times \int_{E_{0}}^{E} \frac{q\left(E^{\prime}\right) \exp \left(-\frac{1}{2} \int_{\epsilon^{\prime}}^{\epsilon} P_{1} d \mathscr{E}_{-}^{\prime \prime} \int_{\epsilon^{\prime}}^{\epsilon} P_{2}^{1 / 2} d \mathscr{E}^{\prime \prime} \mathscr{B} d E^{\prime}\right.}{D^{3 / 4}\left(E^{\prime}\right) a^{1 / 4}\left(\mathscr{E}^{\prime}\right)}
\end{aligned}
$$


where $P_{2}=-a(\mathscr{E}) / D(E)$ and

$$
a(\mathscr{E})=\frac{1}{\tau}+\frac{d A}{d E}-\frac{d^{2} D}{d E^{2}} .
$$

If for simplicity (but not necessary) we consider an average value of $a(\mathscr{E})$ between $\mathscr{E}^{\prime}$ and $\mathscr{E}$, namely $a$, the previous spectrum reduces to

$$
\begin{array}{r}
N(E)=\frac{D^{1 / 4}(E)}{2 a^{1 / 2}} \int_{E_{0}}^{E} \frac{q\left(E^{\prime}\right) \exp \left(-R_{1}-2 a^{1 / 2} R_{2}\right) d E^{\prime}}{D^{3 / 4}\left(E^{\prime}\right)} \\
\text { (particle/energy) } .
\end{array}
$$

It can be seen that this expression may also be obtained by making $\mathrm{t} \rightarrow \infty$ in equation (8).

By substitution of specific rates $A(E)$ and $D(E)$, and an injection spectrum $q(E)$ in equations ( 8$)$ and (11), we obtain analytical expressions, as for instance, for a Fermi process with monoenergetic types injection, which appears in equations (A1) and (A2) in Appendix A, respectively.

\subsection{Incomplete (Systematic) Solution \\ 4.2.1. Analytical Time-dependent Solution}

When in equation (1) the term of energy diffusion, $\left\langle d E^{2} /\right.$ $d t\rangle=0$, the solution is

$$
\begin{aligned}
& N(E, t)=q_{t=0}\left(E_{i}\right)\left[A\left(E_{i}\right) / A(E)\right] e^{-t / \tau} \\
& +A^{-1}(E) \int_{E_{i}}^{E} q\left(E^{\prime}\right) e^{-R_{5}} d E^{\prime} \quad \text { (particle/energy), }
\end{aligned}
$$

where $R_{5}=\tau^{-1} \int_{E^{\prime}}^{E} A^{-1}\left(E^{\prime \prime}\right) d E^{\prime \prime}$, and $E_{i}$ is obtained from $t=$ $\int_{E_{i}}^{E} \frac{d E^{\prime}}{A\left(E^{\prime}\right)}$ with $E_{0} \leq E_{i} \leq E^{\prime} \leq E$.

\section{4,2.2. Analytical Stationary Solution}

In this particular case, when $(\partial N / \partial t)=0$, the solution is

$$
N(E)=A^{-1}(E) \int_{E_{0}}^{E} q\left(E^{\prime}\right) e^{-R_{s}} d E^{\prime} \text { (particle/energy) }
$$

Solution (13) may be obtained by making $t \rightarrow \infty$ in equation (12). Both solutions (12) and (13) have been previously reported by Melrose \& Brown (1976) and Melrose (1980).

In Appendix A we illustrate the application of the analytical spectra (8)-(13) for the specific case of Fermi-type acceleration with monoenergetic injection. It should be noted that these spectra correspond to the particular case of $n=2$ (Fermilike acceleration). However, the WKBJ method is powerful enough to derive analytical solutions for any value of $n$. In Appendix $\mathrm{D}$ we give the general solution valid for any $n$, in the steady-state case, and we will give the corresponding solution for the time-dependent case elsewhere.

\subsection{The Injection Spectra $q(E)$}

\subsubsection{Monoenergetic Injection}

Assuming that all particles entering into the acceleration process begin with the same energy $E_{0}$, the injection spectrum may be described by the following expression:

$$
\left.q(E)=. V \delta\left(E-E_{0}\right) \quad \text { (particles of energy } E_{0}\right)
$$

\subsubsection{Thermal Injection}

If acceleration initiates from thermal energy, the injection spectrum takes the following form:

$$
q(E)=. \vee 2 \pi E^{1 / 2} e^{-E / k T} /(\pi k T)^{3 / 2} \text { (particle/energy), }
$$

where $k$ is the Boltzmann constant and $T$ is the temperature.

\subsubsection{Injection by DC Fields from a Magnetic Neutral Current Sheet}

If the injection takes place during the process of magnetic reconnection in a magnetic neutral current sheet (MNCS), therefore according to Pérez-Peraza et al. $(1977,1978)$ the particle spectrum from a MNCS topology as that given by Priest (1973) is

$$
\begin{aligned}
q(E)= & 1.27 \times 10^{4} \cdot V^{\circ} B\left(m / n_{e}\right)^{1 / 2}\left(E_{c}^{1 / 4} / E^{3 / 4}\right) \\
& \times \exp \left[-1.12\left(E / E_{c}\right)^{3 / 4}\right] \quad(\text { particle/energy })
\end{aligned}
$$

where $E_{c}=\left(e L B V_{d} m^{1 / 2} / 2 c\right)^{2 / 3}, n_{e}=$ number particle density, $L=$ length of the neutral current sheet, $m$ and $e$ are the particle mass and electronic charge, respectively, $V_{d}=0.057 v_{a}$ is the diffusion velocity between matter and field lines, $v_{a}$ is the Alfvén velocity, $c$ is the speed of light and .1 is the number of particles in the diffusion volume that are accelerated by the electric field $E \simeq(1 / c) V_{d} B$.

The parameter $r$ in $q(E)$, within the frame of equations (8)-(13), indicates the number of particles injected per unit time (continuous injection) and has been denoted by $q_{0}$ (particles $/ \mathrm{s})$ in the spectra of the Appendixes. When . $v$ appears in $q_{t=0}$ within the frame of the first term of equations (8) and (12), it denotes the total number of impulsively injected particles in a pulse at $t=0$ (instantaneous injection) and has been denoted by $N_{0}$ (particles) in the spectra of the Appendixes. In the case of monoenergetic injection. $V^{\prime}\left(q_{0}, N_{0}\right)$ refers to particles of energy $E_{0}$. For thermal and (MNCS) injection, $v$ refers to particles of $E \geq E_{0}$. For evaluations of . 1 from equations (14)-(16), we have normalized in $E=E_{0}$ as follows: $q_{t=0}=1$ particles $/ \mathrm{MeV}$ and $q=1$ particle $/ \mathrm{s} \mathrm{MeV}$. The corresponding spectra (eqs. [8]-[13]) are shown in Figures $1-12$.

\section{RESULTS}

In order to test the accuracy level of the analytical solutions obtained from the WKBJ method, we first proceed to compare them to the existing outstanding descriptions of particle spectra: (1) the analytical expressions of the equilibrium spectra in the nonrelativistic and in the ultrarelativistic ranges (Ramaty 1979), illustrated in Figure 1 (dashed lines) and Figure 2 ( solid lines), respectively, (2) A Monte Carlo simulation up to the transrelativistic range, shown with crosses in Figure 1 (Miller et al. 1987), and (3) Numerical calculations of particle spectra through the entire energy range, illustrated with solid lines in Figures 1 and 3 (Miller et al. 1990). Our equilibrium spectrum (eq. [11]) is represented by closed circles in Figure 1, where it can be seen that it coincides with the numerical spectrum. In 
Figure 2 two asymptotic analytical spectra in the ultrarelativistic range are shown for $E \gg m c^{2}$ (solid lines), and for $\beta=1$ according to equation (B1) of Appendix B; also, the equilibrium spectrum given in equation ( 11 ) is shown with open circles. It can be appreciated that the asymptotic solution with $E \gg m c^{2}$ fits quite correctly the overall solution with the WKBJ method, becoming identical for injection energy values $E_{0} \geq$ $10^{5} \mathrm{MeV}$, whereas the solution with $\beta=1$ requires much higher values of $E_{0}$ to approach the overall WKBJ solution. Figure 3 shows time-dependent proton spectra; the open circles represent continuous injection (second term of eq. [8]). The analysis of Figures (1)-(3) indicates the high level of accuracy of the WKBJ method to solve the transport equation (1).

Therefore, having a high confidence in the analytical solutions given in $\$ 4$ for the representation of the energy spectra through the entire energy range, the next step is to perform a quantitative evaluation of the contribution of each energy change rate, the diffusive and the systematic one, to the formation of the actual energy spectrum. This has been done by confrontation of the so-called complete and incomplete solutions in the previous section. For the sake of generality in this study we have chosen two different kinds of turbulence, providing different acceleration rates (eqs. [2]-[5]). For MHD turbulence (Fermi-type acceleration) we have used values of the acceleration efficiency in the range $0.005 \mathrm{~s}^{-1} \leq \alpha \leq 0.8 \mathrm{~s}^{-1}$, containing the most common values usually employed in the literature for coronal flare levels $\alpha \sim(0.03-0.05) \mathrm{s}^{-1}$. The acceleration parameter for Langmuir turbulence has been calculated with equation (6). It must be emphasized that the values of $K$ corresponding to a coronal flare $\left(n_{e} \sim 10^{9} \mathrm{~cm}^{-3}\right.$, $\left.T \sim 10^{7}\right)$ are of the order of $K \sim 0.13\left(\mathrm{MeV}^{2} \mathrm{~s}^{-1}\right)$, so that particle spectra calculated with such a low value fall off very fast, making impossible any kind of study within the context of solar particle energies. This indicates that according to our treatment of the evolution equation and the acceleration rates, Langmuir turbulence is not able to produce flare particles at coronal heights. Hence, for the goal of our work we have used values of $K \geq 3 \times 10^{3}\left(\mathrm{MeV}^{2} \mathrm{~s}^{-1}\right)$, corresponding to densities $n_{e} \geq 1.7 \times 10^{11}$ and temperatures $T \leq 2.0 \times 10^{5}$, that is, the low chromospheric basis of the flare. However, it should be noted that such conditions refer to the acceleration region, whereas the injection region corresponds to the hot coronal levels of the flare, where $T \geq 5 \times 10^{6}$ (Figures $8-11$ ), otherwise the thermal spectrum for super-Alfvénic speeds would produce an acceleration spectrum that would drop off drastically fast.

Therefore, whereas in the case of acceleration with (MNCS) injection, particles are injected upwards from below $\left(n_{e} \geq 10^{11}\right.$ $\mathrm{cm}^{-3}$, in the specific case of acceleration by Langmuir turbulence with thermal injection, particles are injected downwards from above.

For the mean confinement time we have used a wide range containing the typical values found in the literature: $0.05 \mathrm{~s} \leq$ $\tau \leq 1 \mathrm{~s}$. Throughout Figures 4-13 the complete and the incomplete (systematic) solutions of equation (1) are shown with solid and dashed lines, respectively. Each set of two curves is denoted by the letters $a, b$, or $c$, according to different values of the acceleration parameters. Figures 4 and 6 show the equilibrium spectrum for acceleration with MHD and Langmuir turbulence, respectively, when the monoenergetic injection is con- sidered. Figures 5 and 7 show the time-dependent spectrum for MHD and Langmuir acceleration, respectively, with monoenergetic injection. Figures 8 and 10 show the equilibrium spectrum for MHD and Langmuir acceleration, respectively, with thermal injection. Figures 9 and 11 show the time-dependent spectrum for MHD and Langmuir acceleration, respectively, with thermal injection. Figures 12 and 13 show the equilibrium and time-dependent spectra, respectively, for MHD acceleration with (MNCS) injection.

The analysis of our results indicates that the effect of neglecting energy diffusion in the transport equation (1) is translated into a substantial modification of the real particle spectra (complete solution) in the magnitude of flux intensity, as well as in the shape of the spectrum. The behavior of such modification shows the following general tendencies (Figures 4-13):

I. An overproduction of the particle flux over all the energy range of solar particles, relative to the real flux intensity (complete solution ).

II. A depletion of the particle flux over all the energy range of solar particles, relative to the real flux intensity (complete solution ).

III. An overproduction of particle flux up to some energy $E^{*}$, followed by a depletion of flux intensity relative to the real flux at $E>E^{*}$.

IV. A depletion of particle flux up to some energy $E^{*}$, followed by an overproduction of flux intensity relative to the real flux at $E>E^{*}$.

V. A depletion (or overproduction) of particle flux up to some energy $E^{*}$, followed by an overproduction (or depletion) of flux intensity in the range $E^{*}<E<E^{* *}$, and followed by a depletion (or overproduction) relative to the real flux intensity at $E>E^{*}$.

Depending on the set of parameters $\left(\alpha, \tau, E_{0}, t, T\right)$ for stochastic acceleration by MHD turbulence, or the set $\left(K, \tau, E_{0}\right.$, $t, T$ ) for stochastic acceleration by Langmuir turbulence, the relationship between the real particle spectra and the incomplete (systematic) spectra follows any of these five tendencies, whatever the kind of injection considered. The deviations among the complete and incomplete spectra are highly sensible to the parameters $\alpha$ (or $K$ ) and $E_{0}$, but only slightly dependent on $\tau$ and $T$. The behavior of the previous tendencies with the time-dependent spectra approaches that of the stationary spectra at a time $t^{*}$, which is usually in the order of $2-5 \mathrm{~s}$, so that the stationary case may be treated as a particular case of the time-dependent situation as $t \rightarrow \infty$. The separation between both solutions, the complete and the incomplete, increases with energy after the cross point $E^{*}$ or $E^{* *}$, so that if this occurs at the highest energy of the illustrated spectra, such an increase cannot be appreciated. As an example of the behavior of the deviations of the incomplete solution relative to the complete solution, we have summarized in Table 1 the tendencies for one of the cases treated in this work. From Table 1 we can infer, for instance, that if we were concerned with protons in the transrelativistic or ultrarelativistic energy range, during the very first seconds of the acceleration phenomenon, we would be overestimating particle flux at those energies if $E_{0}>$ $0.2 \mathrm{MeV}$ (or even in the nonrelativistic energy range if $E_{0} \leq 0.2$ $\mathrm{MeV}$ ) by solving the transport equation without energy spread. On the other hand, for longer times (or in a stationary situation) with $\alpha \leq 0.05 \mathrm{~s}^{-1}$, we would be overestimating the 
TABLE 1

TENDENCIES OF THE RELATIVE BeHAVIOR OF ENERGY SPECTRA OF PROTONS WITH AND WITHOUT ENERGY SPREAD ${ }^{a}$

\begin{tabular}{|c|c|c|}
\hline $\begin{array}{c}\alpha \\
\left(s^{-1}\right)\end{array}$ & $\begin{array}{l}\text { Energy } \\
(\mathrm{MeV})\end{array}$ & Tendency \\
\hline \multicolumn{3}{|c|}{ Time-dependent Spectra $(t<5 \mathrm{~s})$} \\
\hline$(0.005-0.8) \ldots \ldots$ & $\begin{array}{l}E \leq E_{0} \\
E>E_{0}\end{array}$ & $\begin{array}{l}\text { IV } \\
\text { I }\end{array}$ \\
\hline \multicolumn{3}{|c|}{ Steady-State Spectra } \\
\hline$(0.005-0.04) \ldots \ldots$ & $\begin{array}{l}E \leq E_{0} \\
E>E_{0}\end{array}$ & $\begin{array}{l}\text { III } \\
\text { IV }\end{array}$ \\
\hline$(0.05-0.3) \ldots \ldots$ & $\begin{array}{l}E \leq E_{0} \\
E>E_{0}\end{array}$ & $\begin{array}{l}\text { IV } \\
\text { I, II, V }\end{array}$ \\
\hline$(0.4-0.8) \ldots \ldots \ldots$ & $\begin{array}{l}E \leq E_{0} \\
E>E_{0}\end{array}$ & $\begin{array}{l}\text { IV } \\
\text { II }\end{array}$ \\
\hline
\end{tabular}

a For Fermi-type acceleration with monoenergetic injection: $E_{0}=0.2 \mathrm{MeV}, \tau=(0.05-1.0) \mathrm{s}$.

proton flux intensity at high energies if $E_{0} \leq 0.2 \mathrm{MeV}$, and underestimating it if $E_{0}>0.2 \mathrm{MeV}$, and conversely in the nonrelativistic range, respectively. However, when $\alpha \geq 0.4 \mathrm{~s}^{-1}$ in this stationary situation, we would be underestimating the flux intensity at any proton energy if $E_{0}>0.2 \mathrm{MeV}$, whereas if $E_{0} \leq 0.2 \mathrm{MeV}$ we would be doing an underestimation in the low-energy range and an overestimation at high energies relative to the actual proton flux intensity. For this particular case of Fermi-type acceleration with monoenergetic injection, we

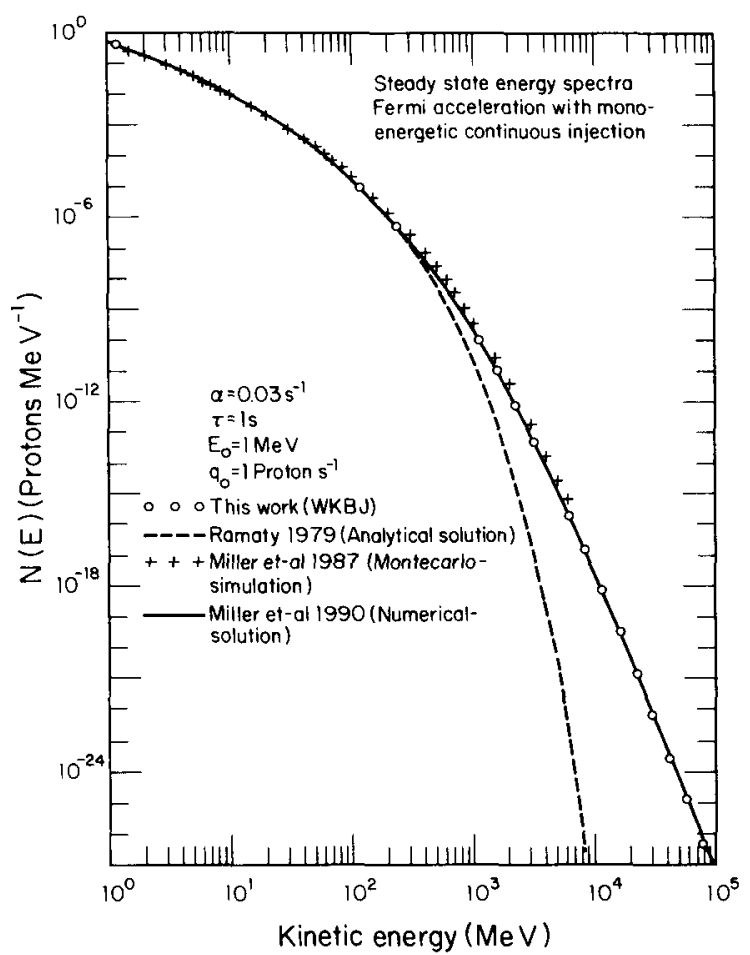

Fig. 1.-Equilibrium spectra for Fermi acceleration with monoenergetic injection.

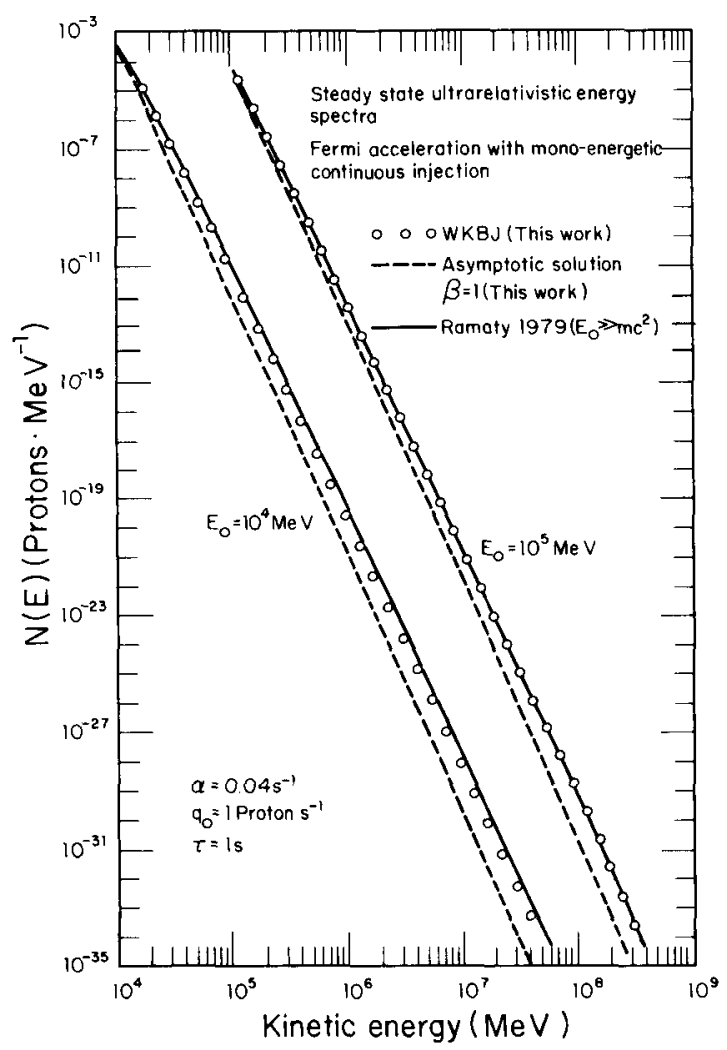

FIG. 2.-Ultrarelativistic equilibrium spectra for Fermi acceleration with monoenergetic injection.

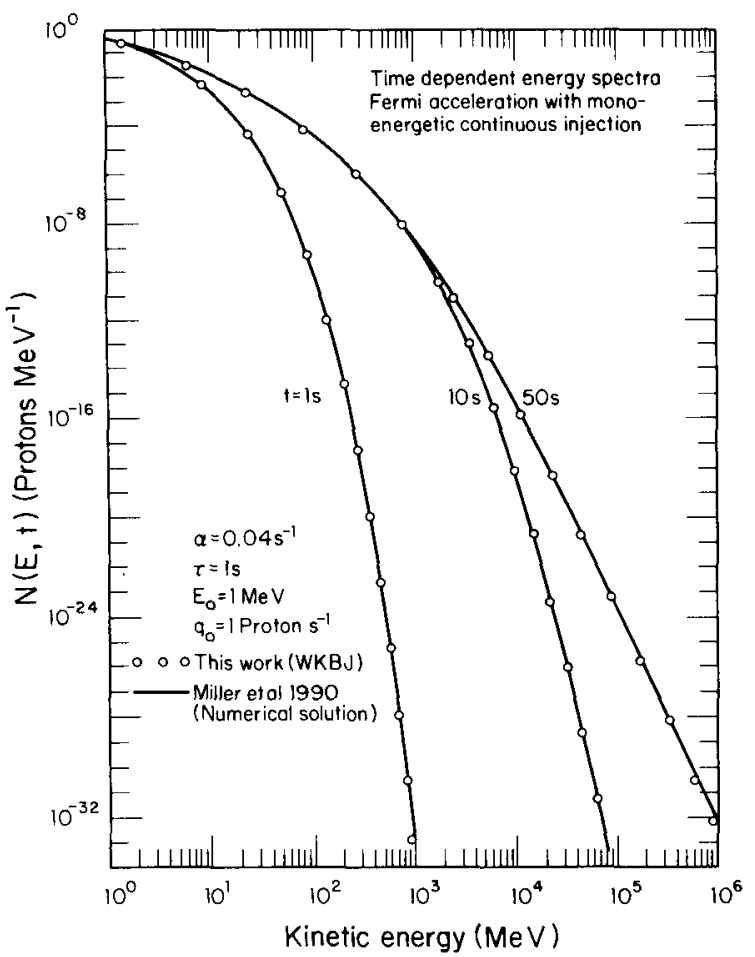

FIG. 3.-Time-dependent energy spectra for Fermi acceleration with monoenergetic injection. 


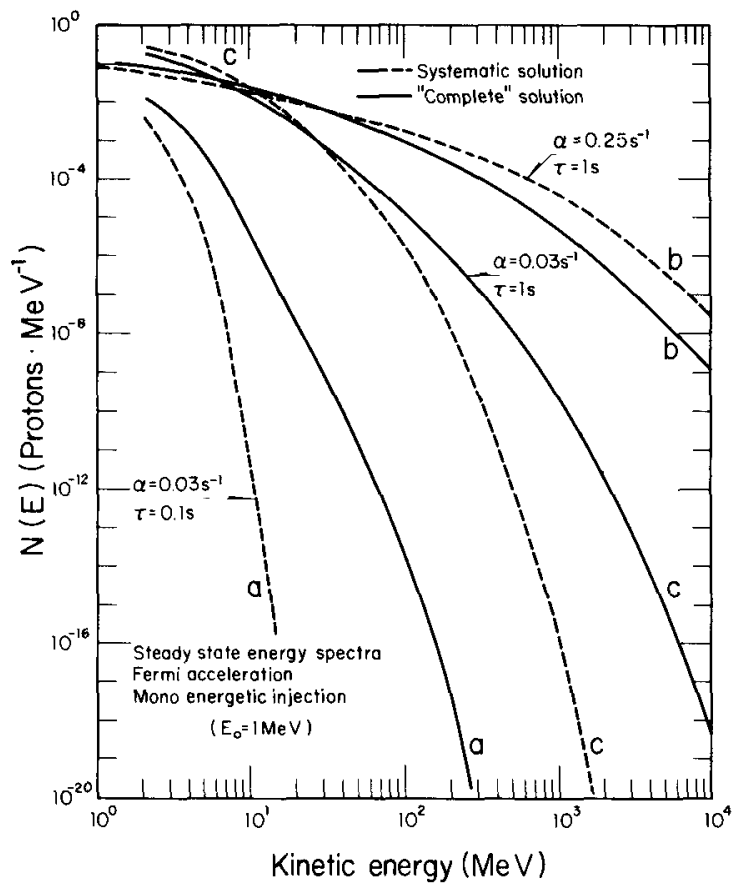

FIG. 4.-Steady-state solutions of eq. (1) by the WKBJ method, for MHD turbulent acceleration with monoenergetic injection.

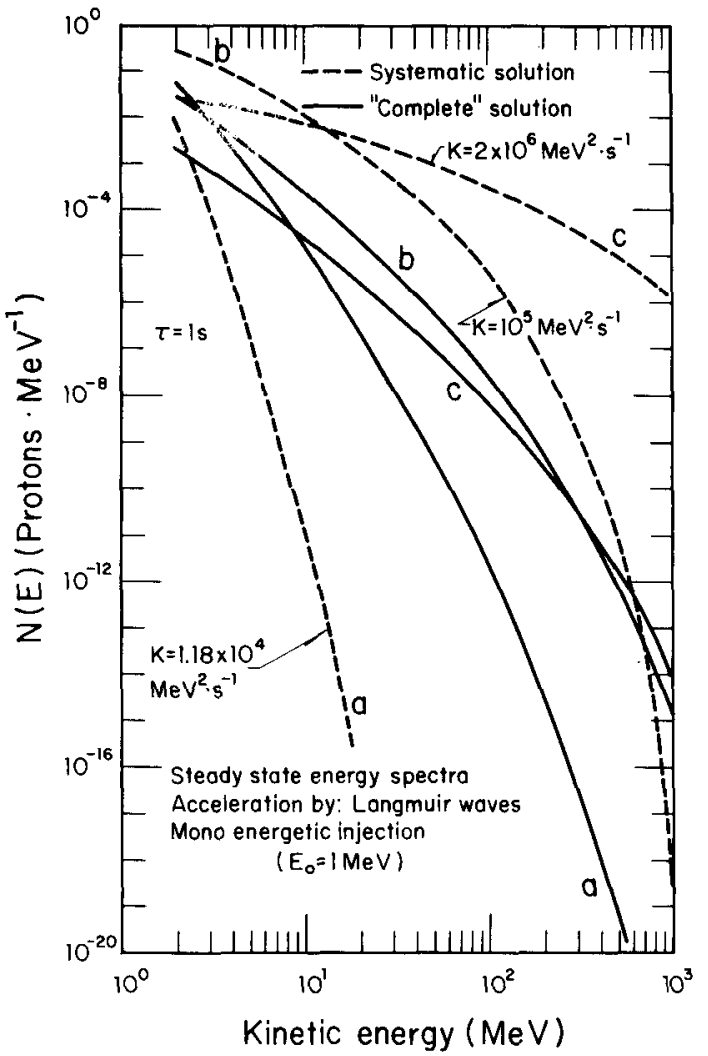

FIG. 6.-Steady-state solutions of eq. (1) by the WKBJ method, for Langmuir turbulent acceleration with monoenergetic injection.

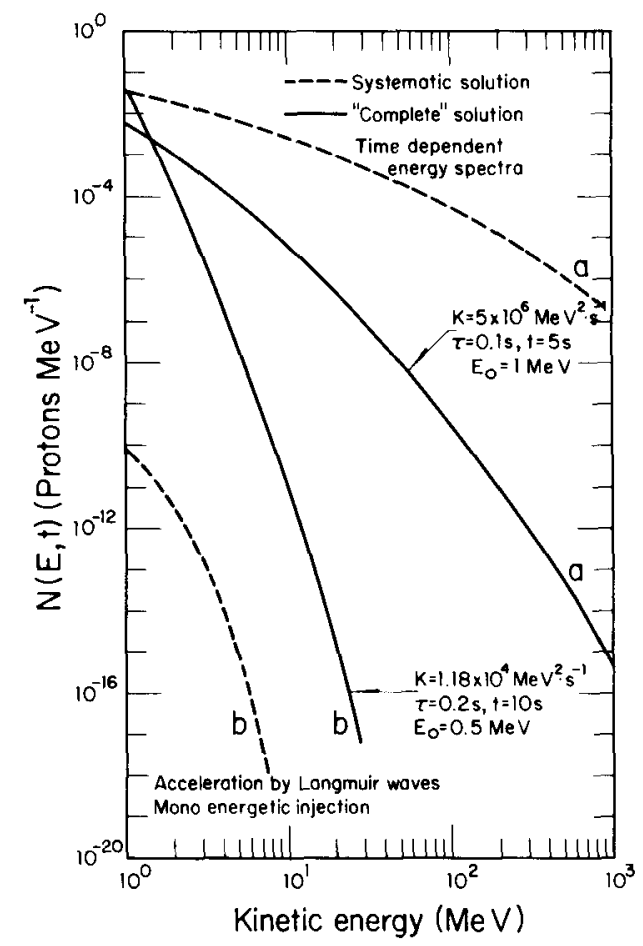

FIG. 7.-Time-dependent solutions of eq. (1) by the WKBJ method, for Langmuir turbulent acceleration with monoenergetic injection.
FIG. 5.-Time-dependent solutions of eq. (1) by the WKBJ method, for MHD turbulent acceleration with monoenergetic injection.

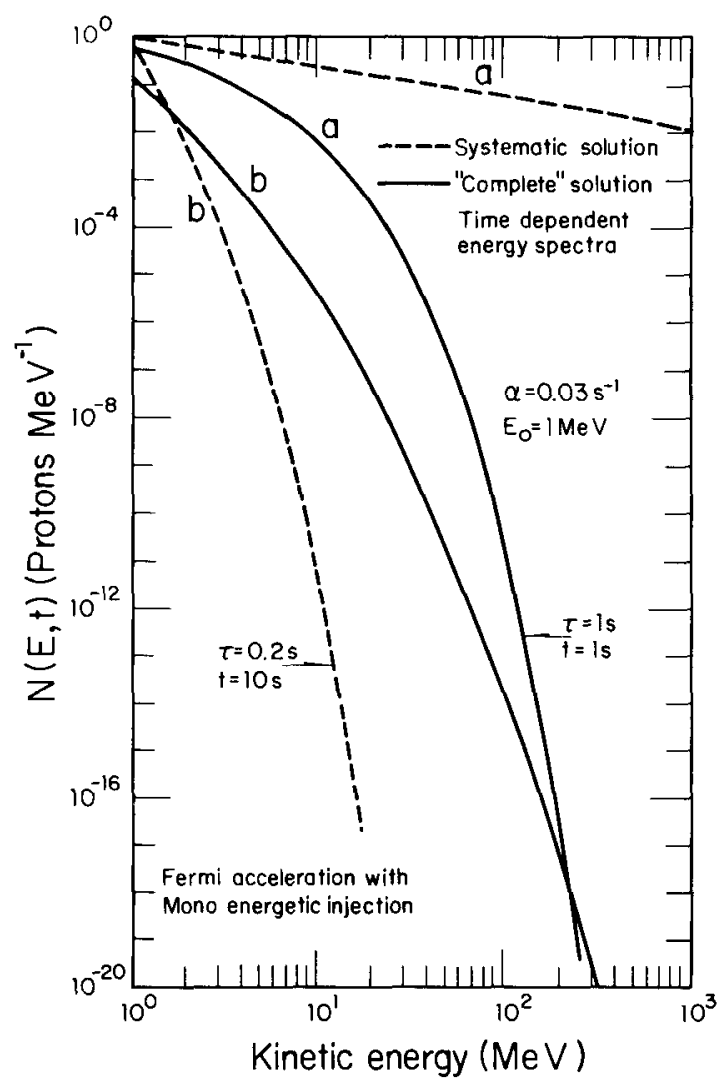




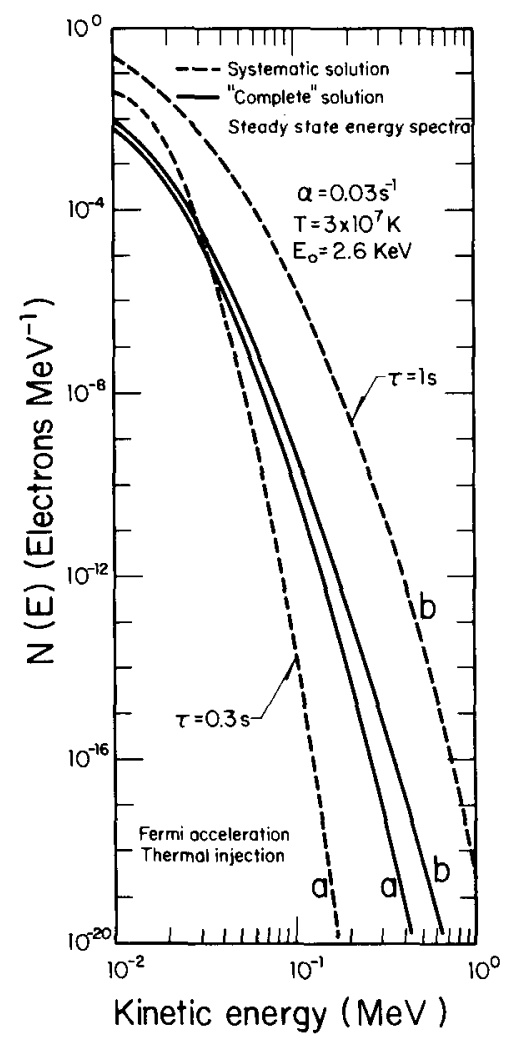

FIG. 8.--Steady-state solutions of eq. (1) by the WKBJ method, for MHD turbulence with thermal injection.

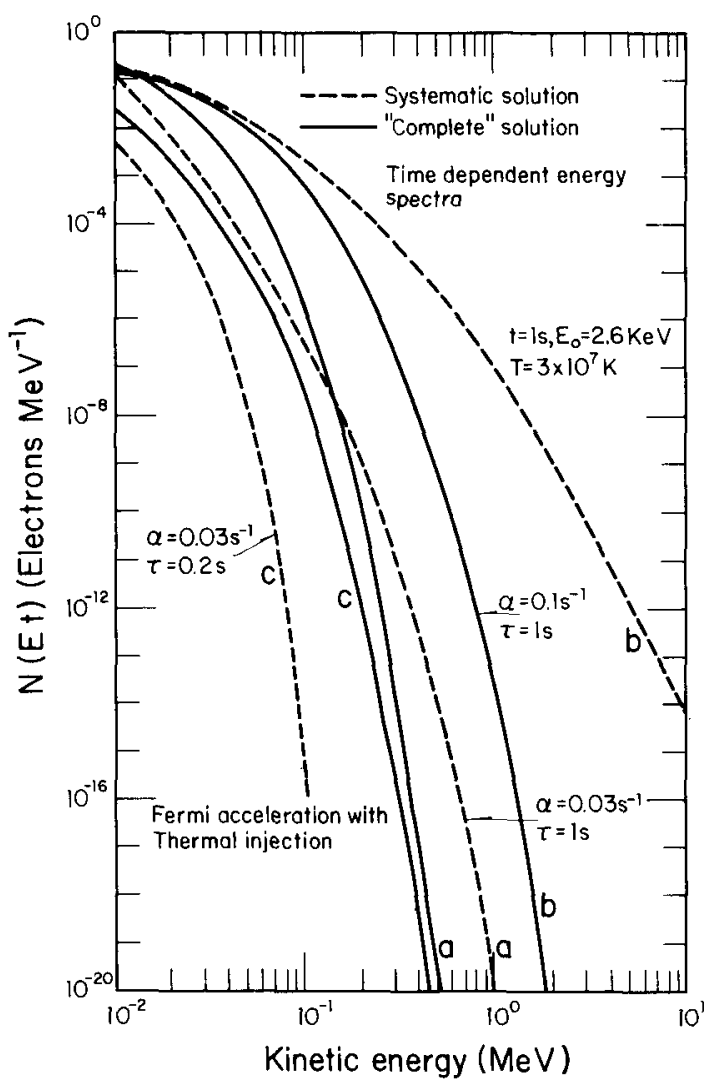

FIG. 9.-Time-dependent solutions of eq. (1) by the WKBJ method, for MHD turbulent acceleration with thermal injection.

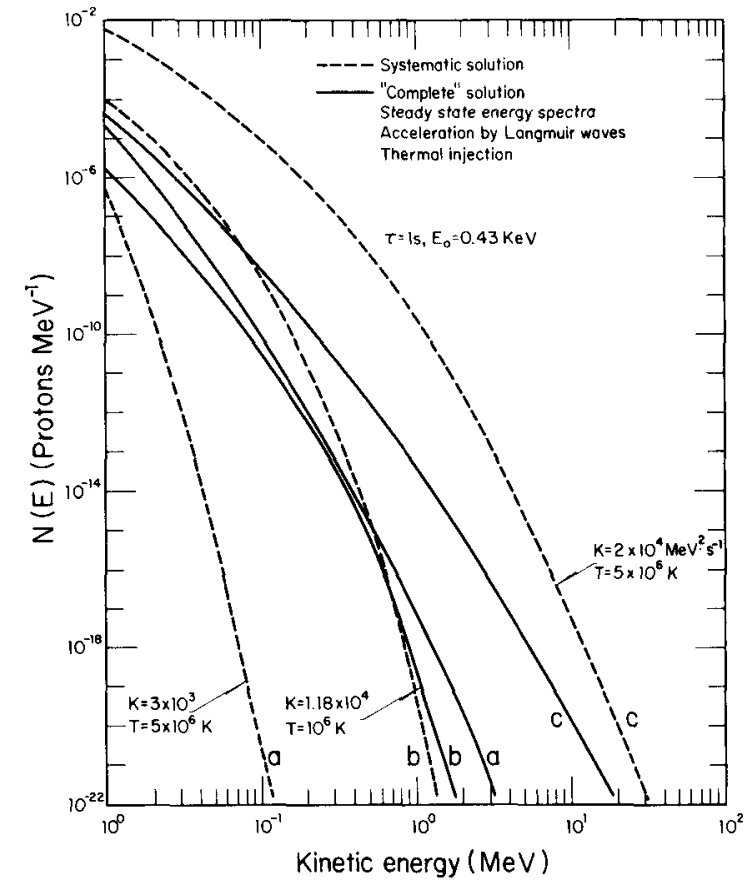

FIG. 10.-Steady-state solutions of eq. (1) by the WKBJ method, for Langmuir turbulent acceleration with thermal injection.

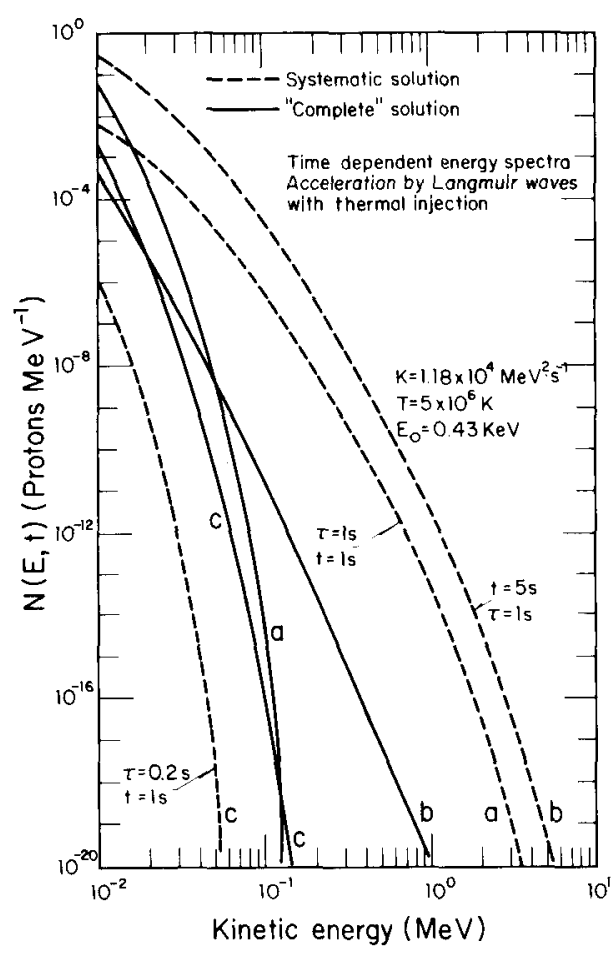

FIG. 11.-Time-dependent solutions of eq. (1) by the WKBJ method, for Langmuir turbulent acceleration with monoenergetic injection. 


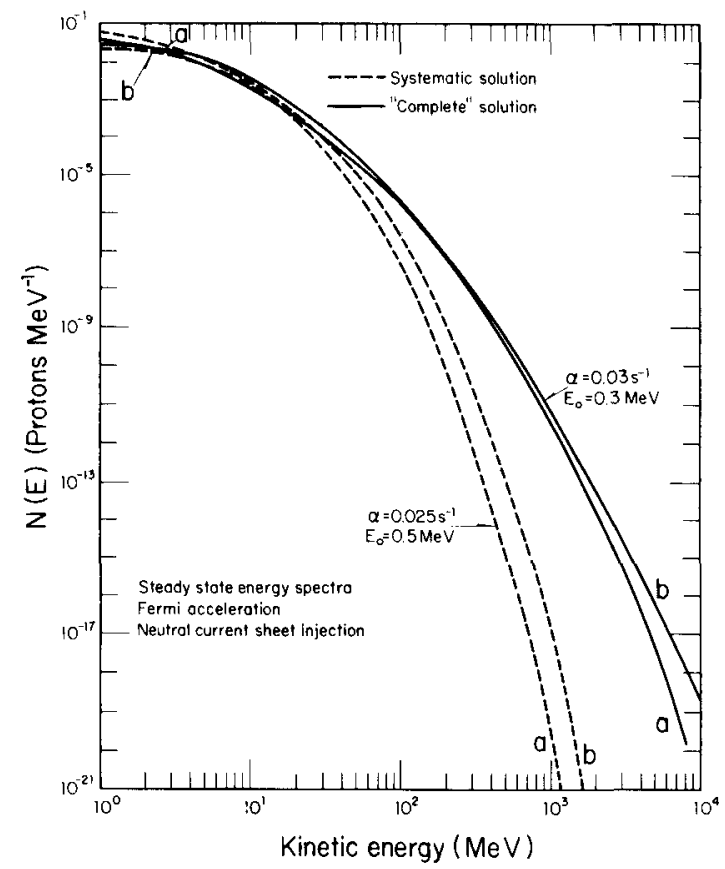

FIG. 12.-Steady-state solutions of eq. (1) by the WKBJ method, for MHD turbulent acceleration with (MNCS) injection.

found that the conditions under which the incomplete solution is within one order of magnitude relative to the complete solution may be found in the range $0.08<\alpha<0.3 \mathrm{~s}^{-1}$, mainly when $E_{0}>0.2 \mathrm{MeV}$.

\section{DISCUSSION}

Conceptually, diffusion in energy space is similar to spatial diffusion, where particle encounters with em inhomogeneities produce changes in the direction they would have followed

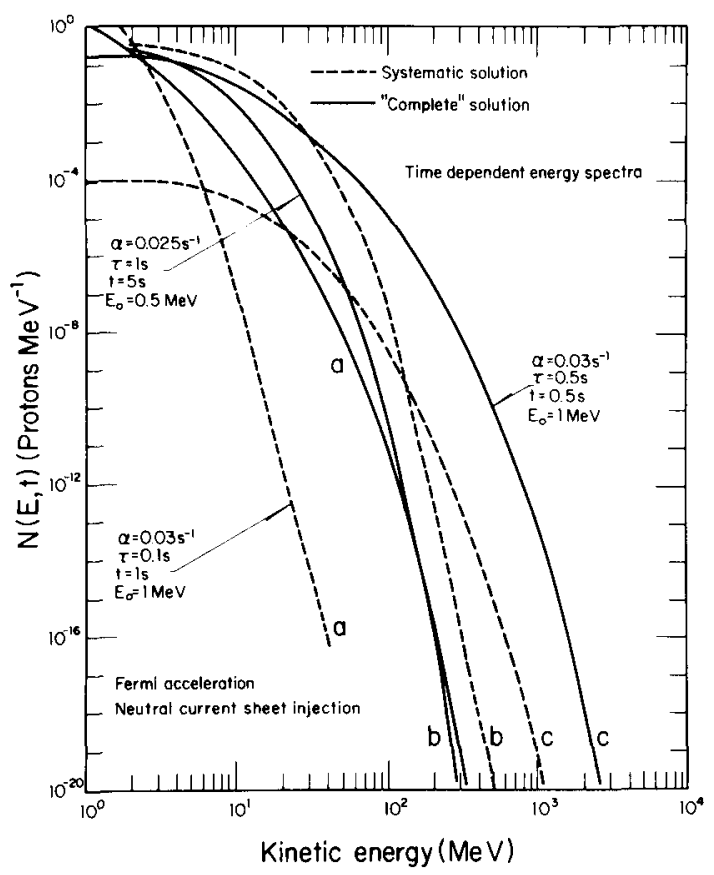

FIG. 13.-Time-dependent solutions of eq. (1) by the WKBJ method, for MHD turbulent acceleration with (MNCS) injection. along the background magnetic field lines if no inhomogeneities had been present. Analogously, diffusion in energy may be seen as a change of direction in the energy change process, relative to the deterministic energy gain process which characterizes a systematic acceleration rate in the absence of energy spread. Actually, in this work diffusion in energy implies the escape of particles from some energy bands (depletion), and their accumulation in other energy bands (overproduction) relative to the distribution from a unidirectional systematic acceleration rate.

In stochastic acceleration, particles interchange energy randomly by a resonant interaction process where the electric fields associated with the MHD or the electrostatic waves may energize particles which have velocities so close to the phase velocity $v_{\phi}$ of waves that at time $t$ have not yet gone a half-wavelength relative to the wave (Chen 1974)

$$
\left|v-v_{\phi}\right| t<\lambda / 2 \text {. }
$$

So, as time goes on, the number of resonant particles decreases, since an increasing number will have shifted more than $\lambda / 2$ from their original positions, that is, they will have changed their resonant band as acceleration goes on. Particles within the resonant interval defined in equation (17) going slower than the wave gain energy from the wave, and those going faster than the wave cede energy to the wave. The equivalent situation in the classic Fermi acceleration process are the socalled head-on and catch-up collisions. For the kind of injection spectra considered in this work the particle number $N_{1}=$ $N\left(v \leq v_{\phi}\right)$ is higher than $N_{2}=N\left(v>v_{\phi}\right)$ at $E \geq E_{0}$ although clearly the proportion $N_{1} / N_{2}$ is strongly energy-dependent. The width of the resonant interval $\left|v_{\phi}-v\right|$ and the energy dependence of the proportion $N_{1} / N_{2}$ depend on the correlation between the wave velocity and particle velocity spectra: if the turbulence spectrum does not contain waves whose velocities lie near the velocity of the bulk of particles, the systematic acceleration rate falls off, and only the dispersive energy change rate counts. Conversely, if the velocity of the bulk of waves in the turbulence spectrum lies near the velocity of the bulk of particles, the diffusive energy change rate falls off relative to the systematic acceleration rate. Since the diffusive rate is determined by the dispersion in the average energy gain, the previous statements may be described in terms of statistic variance, by remembering that variance (energy spread in this case) increases as the number of events (resonant interactions in this case) decreases, and conversely, when the bulk of both populations (waves and particles) are well "centered," so that most particles have velocities near the phase velocities of maximum resonance, $\bar{v}_{\phi}$, the number of resonant interactions is high and hence the variance is low. If most of particles have velocities far from the center of maximum resonance $\bar{v}_{\phi}$, then the number of resonant interactions is low, and thus the spread in the energy change rate is high.

Defining $R=\left(N_{1} / N_{2}\right)_{c} /\left(N_{1} / N_{2}\right)_{s}$ as the ratio between the proportion of accelerated particles in the complete spectra relative to the proportion of accelerated particles in the systematic spectra, if $R \gg 1$ or $R \ll 1$ implies that for the specific acceleration parameters employed in our spectra, the bulk of particle velocities lie far from $\bar{v}_{\phi}$, whereas when $R \rightarrow 1$ most of particle velocities are near $\bar{v}_{\phi}$ so to satisfy equation (17). When $R \simeq 1$ the number of resonant interactions is maximum and the dis- 
persive rate is minimum. On the other hand, the fact that $R>1$ in a given energy range implies that for the employed parameters (the acceleration efficiency mainly) there is a shift of $\bar{v}_{\phi}$ to a higher value, so that there are more particles with $v \leq \bar{v}_{\phi}$ in the complete spectrum relative to the systematic spectrum, producing the overproduction shown in Figs. 4-13. Conversely, when $R<1$ there is a shift of $\bar{v}_{\phi}$ to a lower value, so that the number of particles with $v \leq \bar{v}_{\phi}$ in the resonant bands is decreased in the complete spectrum relative to the systematic spectrum, producing the observed depletion.

Since the Kolmogorov turbulent spectrum is an inverse power law and particle spectra are decreasing quasi-exponential functions, wave-particle interactions are more scarce at high energies, with the subsequent increase in variance (diffusion effects) as can be seen in our results.

Diffusion effects decrease as acceleration efficiency increases, because a variation of $\alpha$ in the schema of MHD turbulence entails a direct variation of $\bar{v}_{\phi}$, since $\alpha \propto D(p) \propto\left(\bar{v}_{\phi} /\right.$ $B^{2}$ ) (Achterberg 1981), so that when $\bar{v}_{\phi}$ shifts to a higher value because of an increase in $\alpha$, the number of resonant interactions increases (diffusion decreases) as the number of particles with $v \leq \bar{v}_{\phi}$ increases. In the schema of Langmuir turbulence, an increase of $K$ implies an increase of the strength of the wave electric fields due to changes in the density and temperature of the plasma (equations [6]-[7]). Similarly, since the higher the acceleration efficiency the higher the number of resonant accelerating interactions, thus the rate of energy spread decreases.

Depletion of particles in some energy bands and accumulation in others may be seen from equation (17): as time elapses, the number of resonant particles per resonant band decreases as an increasing number will have shifted more than $\lambda / 2$ from their original positions, going into other resonant bands, while other particles may eventually arrive from contiguous resonant bands.

A quantitative analysis of particles diffusing into and out of resonant energy bands may be performed by the kinematic equation of the evolution of particles in a resonant interval as derived by Canuto et al. (1978). This equation balances the confinement of particles in the resonant interval against their drifting out as particle velocity retreats from the center of maximum resonance $\bar{v}_{\phi}$. Such an analysis is beyond the scope of this work.

\section{CONCLUSIONS}

We have presented analytical particle energy spectra that are valid through the entire energy range of accelerated particles. We have shown that, in contrast with frequent assumptions which regard the contribution of the term of energy diffusion, in the transport equation, as mere fluctuations in the particle flux, actually the basic energy change rate in a stochastic mech- anism is the diffusion in energy, from which a certain average tendency may be isolated and designated as the systematic acceleration rate. Hence, the frequently so-called fluctuational acceleration in the literature may be somewhat misleading in the sense that diffusion is not translated as fluctuations of particle flux over the energy spectrum, but it represents an important modulation that in general may reach several orders of magnitude relative to the average tendency given by the systematic rate. Although Tsytovich (1966) and Melrose (1968, p. 213 ) argue that typically systematic and fluctuating acceleration are of the same order of magnitude, and the latter need only be considered when the former vanishes, we have found by using accurate analytical energy spectra that the real situation is that dispersive acceleration differs from systematic acceleration by several orders of magnitude, as is shown through Figures 4-12, and they become of the same order only for the particular case of very high acceleration efficiency and injection energy threshold values. In fact, we have shown that whatever the situation is during the acceleration, either steady-state or time-dependent, the actual acceleration rate approaches closer to the average tendency of the systematic rate as the parameter of acceleration efficiency increases, which entails a higher rate of resonant interactions and thus a lower dispersive rate. For the particular case of stochastic acceleration of protons by MHD turbulence, the conditions for such minimization of diffusive effects may occur for $\alpha>0.08 \mathrm{~s}^{-1}$ and $E>$ $0.2 \mathrm{MeV}$.

In agreement with Melrose (1980), we have found that particle acceleration by Langmuir turbulence at coronal flare levels is not plausible because the acceleration spectrum drops off very fast at very low energies; in contrast we found here that it may eventually represent a viable acceleration process in denser and cooler layers of the solar atmosphere, as a well-defined energy spectrum may be formed, provided that such a kind of turbulence may exist in chromospheric flares (e.g., Hoyng 1987a, b; Heyvaerts 1981).

We conclude that (1) the so-called second Fokker-Planck coefficient or diffusive energy rate cannot in general be neglected, mainly when a high precision of the acceleration energy spectrum is required, as is the case for calculation of the fluxes of secondary radiation produced at the source level, and (2) the presented analytical spectra are of particular importance to gamma-ray, neutron, and pion production in solar flares, although they are not exclusive, and may be used for particle acceleration and production of secondary radiation at other astrophysical scales. We claim that these results will allow for the association of secondary production to model-dependent acceleration parameters, which in turn might be associated to specific scenarios of energetic particle phenomena at the source level.

\section{APPENDIX A \\ FERMI ACCELERATION SPECTRA WITH MONOENERGETIC INJECTION}

For the complete time-dependent spectrum, from equation (8) with the Fermi-type acceleration rates (eqs. [2]-[3]), we have

$$
\begin{aligned}
N(E, t)=\frac{1}{(4 \pi \alpha / 3)^{1 / 2}} & \left(\beta_{0} / \beta\right)^{1 / 4}\left(\mathscr{E}_{0} / \mathscr{E}_{0}\right)^{1 / 2} \frac{1}{\beta_{0}^{3 / 2} \mathscr{E}_{0}}\left[\left[\left(N_{0} / t^{1 / 2}\right) \exp \left(-a t-3 J^{2} / 4 \alpha t\right)+\left(q_{0} / 2\right)(\pi / a)^{1 / 2}\right.\right. \\
& \left.\times\left\{\left[\operatorname{erf}\left(Z_{1}\right)-1\right] \exp \left(J(3 a / \alpha)^{1 / 2}\right)+\left[\operatorname{erf}\left(z_{2}\right)+1\right] \exp \left(-J(3 a / \alpha)^{1 / 2}\right\}\right]\right] \quad(\text { particle/energy })
\end{aligned}
$$


where $z_{1,2}=(a t)^{1 / 2} \pm(3 a / 4 \alpha t)^{1 / 2} J, a=(\alpha / 3)(\bar{F}+3 / \alpha \tau), \bar{F}=\frac{1}{2}\left[F(\beta)+F\left(\beta_{0}\right)\right], F(\beta)=\beta^{-1}+3 \beta-2 \beta^{3}, F\left(\beta_{0}\right)=\beta_{0}^{-1}+3 \beta_{0}-2 \beta_{0}^{3}$, $J=\tan ^{-1} \beta^{1 / 2}-\tan ^{-1} \beta_{0}^{1 / 2}+0.5 \ln \left[\left(1+\beta^{1 / 2}\right)\left(1-\beta_{0}^{1 / 2}\right)\right] /\left[\left(1-\beta^{1 / 2}\right)\left(1+\beta_{0}^{1 / 2}\right)\right], \beta=\left(\mathscr{E}^{2}-m^{2} c^{4}\right)^{1 / 2} / \mathscr{E}$, and $\beta_{0}=\beta(\mathscr{E})_{E=E_{0}}$.

For the complete stationary spectrum, from equation (3) with the Fermi-type acceleration rates we have

$$
N(E)=\frac{\left(q_{0} / 2\right)\left(\beta_{0} / \beta\right)^{1 / 4}\left(\mathscr{E} / \mathscr{E}_{0}\right)^{1 / 2} \exp (-3 a / \alpha)^{1 / 2} J}{(a \alpha / 3)^{1 / 2} \beta_{0}^{3 / 2} \mathscr{E}_{0}} \quad(\text { particle/energy })
$$

for the incomplete (systematic) time-dependent spectrum, from equation (12) with the Fermi-type acceleration rates we have

$$
N(E, t)=N_{0}\left(\beta_{0} \mathscr{E}_{0} / \beta \mathscr{E}\right) e^{-t / \tau}+\left(3 q_{0} / 4 \alpha \beta \mathscr{E}\right)\left[(\mathscr{E}+\beta \mathscr{E}) /\left(\mathscr{E}_{0}+\beta_{0} \mathscr{E}\right)\right]^{-3 / 4 \alpha \tau} \quad \text { (particle/energy) }
$$

For the incomplete (systematic) stationary spectrum, from equation (13) with the Fermi-type acceleration rates we have

$$
N(E)=\left(3 q_{0} / 4 \alpha \beta \mathscr{E}\right)\left[(\mathscr{E}+\beta \mathscr{E}) /\left(\mathscr{E}_{0}+\beta \mathscr{E}_{0}\right)\right]^{-3 / 4 \alpha \tau} \quad(\text { particle/energy }) .
$$

\section{APPENDIX B}

ULTRA-HIGH-ENERGY ASYMPTOTIC SOLUTION $(\beta=1)$ OF THE TRANSPORT EQUATION FOR FERMI-TYPE ACCELERATION

The complete time-dependent spectrum is

$$
\begin{aligned}
N(E, t)=\frac{1}{(4 \pi \alpha / 3)^{1 / 2} E_{0}} \int_{E_{0}}^{E}\left[\left[q_{t=0}\left(E^{\prime}\right) / t^{1 / 2} \exp (-r t-b / t)+\left[q\left(E^{\prime}\right) / 2\right](\pi / r)^{1 / 2}\right.\right. \\
\\
\left.\left.\quad \times\left\{\left[\operatorname{erf}\left(Z_{1}\right)-1\right] \exp \left(M_{1}\right)+\left[\operatorname{erf}\left(z_{2}\right)+1\right] \exp \left(-M_{1}\right)\right\}\right]\right] d E^{\prime} \quad \text { (particle/energy) }
\end{aligned}
$$

where $r=(\alpha / 3)(2+3 / \alpha \tau), b=\left[\ln \left(E / E^{\prime}\right)\right]^{2} /(4 \alpha / 3), M_{1}=(3 r / \alpha)^{1 / 2} \ln \left(E / E^{\prime}\right)$, and $Z_{1,2}=(r t)^{1 / 2} \pm \ln \left(E / E^{\prime}\right) /(4 \alpha t / 3)^{1 / 2}$. In the specific case of monoenergetic injection, equation (B1) becomes

$$
\begin{aligned}
N(E, t)=\frac{1}{(4 \pi \alpha / 3)^{1 / 2} E_{0}}\left[\left[\left(N_{0} / t^{1 / 2}\right)\right.\right. & \exp (-r t-b / t)+\left(q_{0} / 2\right)(\pi / r)^{1 / 2} \\
& \left.\left.\times\left\{\left[\operatorname{erf}\left(Z_{1}\right)-1\right] \exp \left(M_{2}\right)+\left[\operatorname{erf}\left(Z_{2}\right)+1\right] \exp \left(-M_{2}\right)\right\}\right]\right] \quad \text { (particle/energy) },
\end{aligned}
$$

where $M_{2}$ is the same as $M_{1}$ evaluated in $E^{\prime}=E_{0}$.

The complete stationary spectrum is

$$
N(E)=\frac{1}{2(r \alpha / 3)^{1 / 2} E_{0}} \int_{E_{0}}^{E} q\left(E^{\prime}\right)\left(E / E^{\prime}\right)^{-(3 r / \alpha)^{1 / 2}} d E^{\prime} \quad(\text { particle/energy }) .
$$

This solution may be obtained from equation (B1) in the limit when $t \rightarrow \infty$. In the specific case of monoenergetic injection equation (B2) becomes

$$
N(E)=\left(q_{0} / 2\right)\left[(\alpha r / 3)^{1 / 2} E_{0}\right]^{-1}\left(E / E_{0}\right)^{-(3 r / \alpha)^{1 / 2}} \quad(\text { particle/energy }) .
$$

\section{APPENDIX C}

STEADY-STATE SOLUTION FOR FERMI ACCELERATION WITH VELOCITY-DEPENDENT ESCAPE TIME $(\tau)$ AND MONOENERGETIC INJECTION

Let us assume that escape time is inversely proportional to particle velocity as $\tau^{-1}(E)=\delta \beta$ (where $\delta$ is a constant), so that the parameter $a(\mathscr{E})$ (eq. [10]) for the case of Fermi acceleration may be rewritten as

$$
a(\mathscr{E})=\frac{1}{\tau(E)}+(\alpha / 3)\left(\beta^{-1}+3 \beta-2 \beta^{3}\right) \approx(\delta+\alpha) \beta+(\alpha / 3) \beta^{-1} .
$$

Now, assuming monoenergetic injection, the steady-state spectrum given in equation (11) may be expressed in the following form:

$$
N(E)=\frac{q_{0} \beta_{0} D^{1 / 4}(E)}{2 a^{1 / 4}(\mathscr{E}) a^{1 / 4}\left(\mathscr{E}_{0}\right) \beta D^{3 / 4}\left(E_{0}\right)} \exp \left[-\int_{E_{0}}^{E} P_{2}^{1 / 2} d E^{\prime}\right],
$$


where

$$
\exp \left[-\int_{E_{0}}^{E} P_{2}^{1 / 2} d E^{\prime}\right]=\exp \left[-\int_{E_{0}}^{E}\left[\frac{a(\mathscr{E})}{D(E)}\right]^{1 / 2} d E^{\prime}\right]=\left(\frac{\mathscr{E}+\beta \mathscr{E}}{\mathscr{E}_{0}+\beta_{0} \mathscr{E}_{0}}\right)^{-(\omega+1 / 2 \omega)} \exp \left[-\frac{1}{2 \omega}\left(\beta_{0}^{-1}-\beta^{-1}\right)\right]
$$

so that by introducing the diffusive rate $D(E)$ for Fermi acceleration in equation (C2), we obtain the next analytical spectrum

$$
N(E)=\frac{(q / 2)\left(\beta_{0} / \beta\right)^{1 / 4}\left(\mathscr{E} / \mathscr{E}_{0}\right)^{1 / 2}}{\left[a^{1 / 2}\left(\mathscr{E}_{0}\right) a^{1 / 2}(\mathscr{E})(\alpha / 3)\right]^{1 / 2} \beta_{0}^{3 / 2} \mathscr{E}_{0}}\left(\frac{\mathscr{E}+\beta \mathscr{E}}{\mathscr{E}_{0}+\beta_{0} \mathscr{E}_{0}}\right)^{-(\omega+1 / 2 \omega)} \exp \left[-\frac{1}{2 \omega}\left(\beta_{0}^{-1}-\beta^{-1}\right)\right],
$$

where $\omega=[3(1+\delta / \alpha)]^{1 / 2}$. Note that the coefficient of spectrum (C3) has the same form as the coefficient of the corresponding spectrum when $\tau=$ constant (eq. [A2]), however, spectrum (C3) tends rapidly to an inverse power law, at least in the transrelativistic and ultrarelativistic ranges, since the exponential factor is $\cong 1$. This is interesting in the light of new observational results on solar particle acceleration and secondary radiation produced at the source, in some specific events, requiring that particle spectra behave as a power law over all the energy range (N. Manhavidze 1993, private communication).

\section{APPENDIX D}

\section{STEADY-STATE SOLUTION OF THE TRANSPORT EQUATION FOR ANY POWER $n$ OF THE MOMENTUM} IN THE MOMENTUM DIFFUSION COEFFICIENT

Let us write $D(p)=(\alpha / 3) p^{n} / \beta$, in which case the systematic and diffusive acceleration rates become, respectively,

$$
\begin{aligned}
& A(E)=\frac{1}{p^{2}} \frac{\partial}{\partial p}\left[v p^{2} D(p)\right]=(\alpha / 3)(n+2) c^{2-n} \beta^{n-1} \mathscr{E}^{n-1}, \\
& D(E)=2 v^{2} D(p)=(\alpha / 3) c^{2-n} \beta^{n+1} \mathscr{E}^{n}=(\alpha / 3 \mathscr{E}) c^{2-n}\left(\mathscr{E}^{2}-m^{2} c^{4}\right)^{(n+1) / 2}
\end{aligned}
$$

The parameter $a(\mathscr{E})$ defined in equation $(10)$ becomes

$$
a(\mathscr{E})=\frac{1}{\tau(E)}+(\alpha / 3) c^{2-n} \mathscr{E}^{n-2}\left[(n-1) \beta^{n-3}+(n+1) \beta^{n-1}-2 \beta^{n+1}\right]
$$

By assuming an escape time given by $\tau^{-1}(E)=\delta \beta^{n-1} \mathscr{E}^{n-2}$ the stationary spectrum for any value of $n$ is

$$
\begin{aligned}
N(E)=\frac{\left(q_{0} / 2\right) \beta^{(1 / 4)(n+1)} \mathscr{E}^{n / 4}}{\left.(\alpha / 3)^{1 / 2} c^{(1 / 2)(2-n)} \beta_{0}^{(3 / 4)(n+1)} \mathscr{E}_{0}^{3 n / 4} a^{1 / 4}(\mathscr{E}) a^{1 / 4} \mathscr{E}_{0}\right)}\left(\frac{\mathscr{E}}{\mathscr{E}_{0}}\right) & \\
& \times\left(\frac{\mathscr{E}_{0}-m^{2} c^{4}}{\mathscr{E}-m^{2} c^{4}}\right)^{n / 2}\left(\frac{\mathscr{E}+\beta \mathscr{E}}{\mathscr{E}_{0}+\beta_{0} \mathscr{E}_{0}}\right)^{-(\omega+[(n-1) / 2 \omega])} \exp \left[-\frac{n-1}{2 \omega}\left(\beta_{0}^{-1}-\beta^{-1}\right)\right],
\end{aligned}
$$

where $\omega=\left\{3\left[(1 / 3)(n+1)+\delta / \alpha c^{2-n}\right]\right\}^{1 / 2}$ for this general case.

By introducing in the general steady-state spectrum (D4) the assumption of a Fermi-type mechanism, that is, $n=2$, we obtain immediately the Fermi spectrum given in equation $(\mathrm{C} 3)$, when the escape time is considered velocity-dependent. Obviously, other $n$ values may be used as for instance $n=5 / 3$ or $n=3 / 2$ (the Kolmogorov and Kraichnan values).

\section{REFERENCES}

Achterberg, A. 1981, A\&A, 97, 259

Bai, T. 1986, ApJ, 308, 912

Borosky, J. E., \& Eilek, J. A. 1986, ApJ, 308, 929

Byakov, A. M. 1992, in AIP Conf. Proc. 264, Particle Acceleration in Cosmic Plasmas ( New York: AIP), 221

Cane, H. V., McGuire, R. E., \& von Rosenvinge, T. T. 1986, ApJ, 301,448 Canuto, V., Chou, C. K., \& Facio-Canuto, R. 1978, Fund. Cosmic Phys., 3,221

Chen, F. F. 1974, Introduction to Plasma Physics (New York: Plenum), chap. 7

Cheng, C. C. 1972, Space Sci. Rev., 13, 3

Davis, L. 1956, Phys. Rev., 101, 351

Dung, R., \& Schlickeiser, R. 1990, A\&A, 237, 504

. 1990, A\&A, 240, 537

Eichler, D. 1979, ApJ, 229, 413

Fermi, E. 1949, Phys. Rev., 75, 1169
Gallegos-Cruz, A., \& Pérez-Peraza, J. 1987, Proc. 20th Internat. CosmicRay Conf. (Moscow), 8, 164

- 1991, Proc. 22nd Internat. Cosmic-Ray Conf. (Dublin), 3,

Gallegos-Cruz, A., Pérez-Peraza, J., Miroschnichenko, L. I., \& Vashenyuk, E. V. 1993, Adv. Space Res., 13, 187

Ginzburg, V. L., \& Syrovatskii, L. I. 1964, The Origin of Cosmic Rays (New York: Pergamon)

Heristchi, Dj., Trottet, G., \& Pérez-Peraza, J. 1976, Sol. Phys., 49, 151 Heyvaerts, J. 1981, in Solar Flare Magnetohydrodynamics, ed. E. R. Priest (New York: Gordon \& Breach), 429

Hoyng P. 1978a, A\&A, 55, 23

1978b, A\&A, 55, 31

Jones, F. C. 1992, in AIP Conf. Proc. 264, Particle Acceleration in Cosmic Plasmas (New York: AIP), 71

Kulsrud, R. M., \& Ferrari, A. 1971, Ap\&SS, 12, 302

Lacombe, C., \& Manganey, A. 1969, A\&A, 1, 325 
MacKinnon, A. L., Brown J. C., Trottet, G., \& Vilmer, N. 1983, A\&A, 119,297

Melrose, D. B. 1968, Ap\&SS, 2, 71

. 1980, Plasma Astrophysics, vol. 2 (New York: Gordon \& Breach), chap. 8

1986, Instabilities in Space and Laboratory Plasmas (Cambridge: Cambridge Univ. Press), 233

Melrose, D. B., \& Brown, J. C. 1976, MNRAS, 176, 15

Miller, J, A. 1991, ApJ, 376, 342

Miller, J. A., Guessoum, N., \& Ramaty, R. 1990, ApJ, 361, 701

Miller, J. A., Ramaty, R., \& Murphy, R. J. 1987, Proc. 20th Internat. Cosmic-Ray Conf. (Moscow) , 3, 33

Parker, E. N., \& Tidman, D. A. 1958, Phys. Rev., 11, 1206

Pérez-Peraza, J. 1986, Space Sci. Rev., 44, 91

Pérez-Peraza, J., \& Gallegos-Cruz, A. 1994, Geofís. Internat., 33

Pérez-Peraza, J., Gálvez, M., \& Lara, R. 1977, Space Res., 18

1978, Proc. 15th Internat. Cosmic-Ray Conf. (Plodviv), 5, 23

Pikel'ner, S. B., \& Livishts, M. A. 1977. Soviet Astron., 21, 601

Priest, E. R. 1973, ApJ, 181, 227

. 1984, Solar Magnetohydrodynamics (Dordrecht: Reidel)
Ramaty, R. 1979, in AIP Conf. Proc. 56, Particle Acceleration in Astrophysics, ed. J. Arons, C. Max, \& C. McKee (New York: AIP), 135 . 1987, Sol. Phys., 113, 203

Rose, W. K., Beall, J. H. Guillory, J., \& Kaimer, S. 1987, ApJ, 314, 95

Schatzman, E. 1966, in Proc. Les Houches Summer School of Theoretical Physics (New York: Gordon \& Breach), 229

Schlickeiser, R. 1989, ApJ, 336, 243

- 1992, in AIP Conf. Proc. 264, Particle Acceleration in Cosmic Plasmas (New York: AIP), 71

Steinacker, J., \& Miller, J. A. 1992, in AIP Conf. Proc. 264, Particle Acceleration in Cosmic Plasmas (New York: AIP), 235

Stix, T. H. 1975, Nucl. Fusion, 15, 737

Tsytovich, V. N. 1966, Soviet Phys. Uspekhi, 9, 370

. 1970, Non-linear Effects in Plasma (New York: Plenum), chap. 6

- 1977, Theory of Turbulent Plasma (New York: Plenum), chap. 5

Tverskoi, B. A. 1967, Soviet Phys. JETP, 25, 317; English trans. 1968, 26, 821

Vilmer, N., Kane, S. R., \& Trottet, G. 1982, A\&A, 108, 306

Vilmer, N., Trottet, G., \& MacKinnon, A. L. 1986, A\&A, 156, 64 